\title{
Measurement tools and antidotes for non-vitamin K oral anticoagulants
}

\author{
Richard C. Becker ${ }^{1}$
}

Published online: 14 September 2015

(c) Springer Science+Business Media New York 2015

Thrombosis is ubiquitous in medicine and society. It can either be protective, stemming blood loss following vascular injury (hemostasis) or life-threatening when occurring without good cause. Thrombosis occurring within an artery is the proximate etiology of myocardial infarction and ischemic stroke, affecting 750,000 and 1 million persons, respectively, each year in the United States. Thrombosis within a vein causes deep vein thrombosis and pulmonary embolism, with up to 600,000 cases yearly and up to a $30 \%$ case fatality rate if untreated.

The burden of thrombosis in health care is the foundation for intense efforts to develop safe, effective and easily manageable alternatives to vitamin K-directed oral anticoagulants. Over the past decade, large- scale clinical trials have convincingly documented their ability to prevent and treat venous and arterial thrombosis and to do so safely in appropriately selected patients.

This special issue of the Journal of Thrombosis and Thrombolysis is dedicated to answering two practical questions being asked by clinicians, hospitals and health care systems around the world. (1) How does one measure the anticoagulant effects of the new or contemporary nonvitamin $\mathrm{K}$ oral anticoagulants? and (2) What are the options for reversing the anticoagulant effects of these new drugs when overt bleeding occurs or it is anticipated because of trauma or a need for an invasive procedure?

We have assembled a group of highly experienced clinicians and scientific investigators to answer these important questions, emphasizing drug pharmacology, the pathobiology of thrombosis in the context of common diseases, disorders and conditions, regulatory pathways for accelerated drug approval, patient decision-making tools, laboratory medicine, best available evidence and good clinical practice.

Our primary objective is to educate and inform those who have dedicated themselves and their life's' work to preventing and treating patients with and those at risk for thrombosis.
Richard C. Becker

richard.becker@uc.edu

1 University of Cincinnati College of Medicine, Cincinnati, $\mathrm{OH}$, USA 